\title{
ON CLOSED IMAGES OF THE SPACE OF IRRATIONALS
}

\section{R. ENGELKING}

In connection with his recent joint work with $\mathrm{A}$. H. Stone on quotients of the space of irrationals (cf. [6]), E. Michael asked whether every complete separable metric space is a continuous image of the irrationals under a closed mapping. The present note contains the positive answer to this question. In fact, we actually prove a more general result which does not assume separability. The proof is based on a refinement of a known lemma (see [5, p. 281], and [2] or [4] for the nonseparable case) that every nonempty closed subset of a metric space $X$ of covering dimension 0 is a retract of $X$. The refinement, which seems to be new even in the separable case, asserts the existence of a closed retraction. Our construction of such a retraction coincides with one given in $\left[9\right.$, Theorem $3^{\prime}$, p. 8$]$ by A. H. Stone, who did not, however, observe that it is closed. We include the details for the sake of completeness. I am grateful to E. Michael for some valuable remarks.

Lemma. For every nonempty closed subset $F$ of a metrizable space $X$ satisfying $\operatorname{dim}(X-F)=0$, there exists a closed continuous function $f: X \rightarrow F$ such that $f \mid F: F \rightarrow F$ is the identity on $F$.

Proof. The function $f$ is defined by a modification of the wellknown retraction of $X$ to $F$ (see [5, p. 281]).

Let $\rho$ be a metric in the space $X$ and let $U_{1}, U_{2}, \cdots$ be a sequence of open-and-closed subsets of $X$ such that

$$
F \subset U_{i} \subset\{x: \rho(x, F)<1 / i\}
$$

(see [1, Theorems 6.2.3 and 6.2.4]). We can suppose, without loss of generality, that the sequence $U_{1}, U_{2}, \ldots$ is decreasing. Let $W_{1}=X-U_{2}$ and $W_{i}=U_{i}-U_{i+1}$ for $i=2,3, \cdots$. We have $X-F$ $=\bigcup_{i-1}^{\infty} W_{i}$. For every $i$ the set $W_{i}$ can be expressed as the union of a family $\mathcal{F}_{i}=\left\{F_{s}\right\}_{s \in S_{i}}$ of disjoint open-and-closed sets whose diameters are smaller than $1 / i$ (see [1, Theorem 7.2.3]); we can suppose that $S_{i} \cap S_{j}=0$ for $i \neq j$. Let $S=\bigcup_{i=1}^{\infty} S_{i}$; it is easy to see that the sets $\left\{F_{s}\right\}_{s \in S}$ are open-and-closed, disjoint and satisfy

(1) If $s_{n} \in S_{i_{n}}$ and $\lim i_{n}=\infty$, then $\lim \delta\left(F_{s_{n}}\right)=0=\lim \rho\left(F, F_{s_{n}}\right)$,

(2) $X-F=\bigcup_{s \in S} F_{s}$.

Received by the editors April 3, 1968. 
For every integer $i$, let $P_{i}$ be a subset of $F$ maximal with respect to the property " $\rho(x, y) \geqq 1 / i$ for any $x, y \in P$." Such a subset exists, because the property is of finite character (see $[1$, p. 21]). Moreover, we have

(3) $P_{i}$ has no accumulation points,

(4) for every $x \in F$ there exists a $p \in P_{i}$ such that $\rho(x, p)<1 / i$.

From (4) it follows that, for every $i$ and $s \in S_{i}$, there exists a point $p_{s} \in P_{i}$ satisfying

(5) $\rho\left(p_{s}, F_{s}\right)<\rho\left(F, F_{s}\right)+2 / i$.

By (2), the formula

$$
\begin{aligned}
& f(x)=x \quad \text { for } x \in F, \\
& =p_{\mathrm{s}} \text { for } x \in F_{\mathrm{s}}
\end{aligned}
$$

defines a function $f: X \rightarrow F$. The family $\left\{F_{s}\right\}_{s \in S}$ being composed of open-and-closed and disjoint sets, the function $f$ is continuous on $\bigcup_{s \in S} F_{s}=X-F$. To prove that $f$ is continuous, it is sufficient to show that

(6) If $x \in F, x=\lim x_{n}$, and $x_{n} \in X-F$, then $x=f(x)=\lim f\left(x_{n}\right)$.

Let $x_{n} \in F_{s_{n}}$, where $s_{n} \in S_{i_{n}}$. As $x=\lim x_{n} \in F$, we have $\lim \rho\left(x_{n}, F\right)$ $=0$ and it follows that $\lim i_{n}=\infty$. Hence (6) is a consequence of the following

(7) If $x_{n} \in F_{s_{n}}, s_{n} \in S_{i_{n}}$, and $\lim i_{n}=\infty$, then $\lim \rho\left(x_{n}, f\left(x_{n}\right)\right)=0$. have

To prove (7) let us note that by (5) and the equality $f\left(x_{n}\right)=p_{s_{n}}$ we

$$
\begin{aligned}
\rho\left(x_{n}, f\left(x_{n}\right)\right) & =\rho\left(x_{n}, p_{s_{n}}\right) \leqq \delta\left(F_{s_{n}}\right)+\rho\left(p_{s_{n}}, F_{s_{n}}\right) \\
& \leqq \delta\left(F_{s_{n}}\right)+\rho\left(F, F_{s_{n}}\right)+2 / i_{n},
\end{aligned}
$$

which by (1) implies (7).

Now we shall prove that $f$ is a closed mapping. Let $A$ be a closed subset of $X$ and let

$$
x \in \overline{f(A)} .
$$

As

$$
\overline{f(A)}=\overline{f(A \cap F)} \cup \overline{f(A-F)}=\overline{A \cap F} \cup \overline{f(A-F)},
$$

we can assume that either

$$
x \in \overline{A \cap F}
$$

or there exists a sequence $x_{1}, x_{2}, \cdots$ of points of $A-F$ such that $x=\lim f\left(x_{n}\right)$. The set $A \cap F$ is closed, so in the first case we have $x \in A \cap F=f(A \cap F) \subset f(A)$. In the second case, let $x_{n} \in F_{s_{n}}$, where 
$s_{n} \in S_{i_{n}}$. We have then $x=\lim p_{s_{n}}$, and (3) implies that $\lim i_{n}=\infty$ or else $x=f\left(x_{n}\right)$ for some $n$. If $x=f\left(x_{n}\right)$, then of course $x \in f(A)$. If $\lim i_{n}$ $=\infty$, then by (7) we have $x=\lim x_{n}$. But $A$ is closed, so $x \in A$, and as $x \in F$ we have $f(x)=x$ and in this case also $x \in f(A)$. This shows that $f(A)$ is closed, and that proves the lemma.

Let us note that, as shown by the example of the real line in the plane, not every retract of an arbitrary metric space $X$ is the image of $X$ under a closed retraction.

For every $\mathfrak{m} \geqq \boldsymbol{N}_{0}$, let $B(\mathfrak{m})$ denote the Baire space of weight $\mathfrak{m}$, i.e. the Cartesian product of $\boldsymbol{\aleph}_{0}$ copies of the discrete space of cardinality m. It is well known (see for example [1, Theorem 7.3.9]) that every metrizable space $X$ satisfying the conditions $\operatorname{dim} X \leqq 0$ and $w(X) \leqq \mathfrak{m}$, where $w(X)$ denotes the weight of $X$, is embeddable in the space $B(\mathfrak{m})$. Looking at the proof of this result one sees that, if $X$ is moreover complete, then its homeomorphic image in $B(\mathfrak{m})$ is closed. Now if $S$ is a subspace of a metrizable space $X$, then $\operatorname{dim} S \leqq \operatorname{dim} X$ (cf. $[1$, Theorem 7.3.3]), and clearly $w(S) \leqq w(X)$. It follows that every $G_{\delta}$-set in $B(\mathfrak{m})$, which must be complete in some metric, is homeomorphic to a closed subset of the space $B(\mathfrak{m})$.

THEOREM. For every nonempty complete metric space $X$ satisfying $w(X) \leqq \mathfrak{m}$, there exists a continuous closed function of the Baire space $B(\mathfrak{m})$ of weight $\mathfrak{m}$ onto $X$.

Proof. By a result of Morita (see [7] or [8]), there exists a perfect mapping $g$ (i.e. a closed mapping such that $g^{-1}(x)$ is compact for any $x \in X)$ defined on a subspace $S$ of $B(\mathfrak{m})$ onto $X$. Since an inverse image of an absolute $G_{\delta}$ under a perfect mapping is an absolute $G_{\delta}$ (see [3]), it follows that $S$ is a $G_{\delta}$-set in $B(\mathfrak{m})$. But by the above remark, $S$ is homeomorphic to a closed subset $S^{\prime}$ of $B(\mathrm{~m})$, so we can assume that $g$ is defined on a closed subset $S^{\prime}$ of $B(\mathfrak{m})$. Composing the retraction of $B(\mathfrak{m})$ onto $S^{\prime}$ satisfying our lemma with $g$, we get a closed function of $B(\mathfrak{m})$ onto $X$.

As $B\left(\boldsymbol{N}_{0}\right)$ is homeomorphic to the space of irrationals, we obtain

Corollary. For every nonempty complete separable metric space $X$, there exists a continuous closed function of the space of irrationals onto $X$.

\section{REFERENCES}

1. R. Engelking, Outline of general topology, North-Holland, Amsterdam, 1968.

2. J. Dugundji, Absolute neighborhood retracts and local connectedness in arbitrary metric spaces, Compositio Math. 13 (1958), 229-246.

3. M. Henriksen and J. R. Isbell, Some properties of compactifications, Duke Math. J. 25 (1958), 83-106. 
4. Y. Kodama, On LCn metric spaces, Proc. Japan Acad. 33 (1957), 79-83.

5. K. Kuratowski, Topology, Vol. 1, Academic Press, New York, 1966.

6. E. Michael and A. H. Stone, Quotients of the space of irrationals, Pacific J. Math. (to appear).

7. K. Morita, A condition for the metrizability of topological spaces and for ndimensionality, Sci. Rep. Tokyo Kyoiku Daigaku Sect. A 5 (1955), 33-36.

8. V. I. Ponomarev, Normal spaces as images of zero-dimensional ones, Dokl. Akad. Nauk SSSR 132 (1960), 1269-1272 = Soviet Math. Dokl. 1 (1960), 774-777.

9. A. H. Stone, Nonseparable Borel sets, Rozprawy Mat. 28 (1962).

UNIVERSITY OF WASHINGTON AND

Institute of Mathematics of the Polish Academy of Sciences 